\title{
Evaluating the effect on seismicity of a hydraulic fracturing trial using Bayesian data analysis
}

\author{
K Jonsson Luossavaara-Kiirunavaara Aktiebolag (LKAB), Sweden \\ J Martinsson Luleå University of Technology and Luossavaara-Kiirunavaara Aktiebolag (LKAB), Sweden
}

\begin{abstract}
During the summer of 2017, a hydraulic fracturing trial was performed in the crown pillar of one of the orebodies in LKAB's mine in Malmberget. Mucking in the orebody beneath the crown pillar recommenced in September 2017, after being shut down since April 2015. The mucking was shut down in 2015 because a number of large seismic events had caused significant disturbances in the nearby town, with vibration up to $35 \mathrm{~mm} / \mathrm{s}$. A research project was started with the final goal to start up production again with less disturbance to the town. Hydraulic fracturing was determined to be an interesting method for improving caveability of the crown pillar, and a trial was set up. The trial consisted of partially fracturing three boreholes drilled from the ground surface into the crown pillar, which is between 550 and $650 \mathrm{~m}$ thick at the trial location. Hydraulic fractures were placed with $2.5 \mathrm{~m}$ spacing at the bottom $50 \mathrm{~m}$ of each hole.

As part of the evaluation of the effectiveness of the trial, the seismicity before and after the hydraulic fracturing trial has been compared using standard Bayesian techniques to infer differences. The analysis show a difference in $b$-value before and after the trial, which is demonstrated by the parameter $\Delta b$ having a positive value. The 95\% high density interval (HDI) of $\Delta b$ excludes the value of zero, which gives confidence in the conclusion. The difference in b-value depends on the choice of magnitude of completeness, so it is recommended that the analysis is repeated with a method using all recorded events, i.e. using the full dataset.
\end{abstract}

Keywords: hydraulic fracturing, seismicity, Bayesian data analysis

\section{Introduction}

Deep mining in hard, brittle rock can be challenging. With high stress and seismicity impacting on mining at LKAB's Kiruna and Malmberget mines, the management of mining-induced seismicity is crucial in order to continue deep mining.

The Malmberget underground mine consists of about 20 orebodies, of which 14 are mined at present. Some orebodies have been mined by surface mining, while mining in others started some distance below the ground surface, leaving a crown pillar. In the Printzsköld orebody, significant seismic events occurred in the crown pillar causing large vibrations in the town of Malmberget, located adjacent to this orebody. Hydraulic fracturing has been successful elsewhere in enhancing caveability (Lowther et al. 2016), so a trial was performed during the summer of 2017, inducing 93 fractures in three holes drilled from the ground surface.

While the hydraulic fracturing was ongoing in dayshift, mucking recommenced in the afternoon shifts in drifts located below the fractured volume. After the trial was completed in late August, mucking was gradually allowed to go up to full production. The working areas both on the ground surface and underground were continuously monitored.

This paper comprises a comparison of the seismicity before and after the hydraulic fracturing trial to determine if the procedure has changed the properties of the rock mass in the crown pillar. 


\section{Background and description of Bayesian model}

Analysing seismic data involves handling large amounts of data, and statistical methods are well-suited for comparing two large datasets with each other. In this study, Bayesian data analysis was chosen over more traditional methods since it allows modelling of the posterior distribution of several parameters and gives possibilities for richer inference. It is also possible to account for the uncertainty or noise in the analysis. Kruschke (2015) has defined the five basic steps of Bayesian analysis as identify the data, define a descriptive model, specify a prior, compute the posterior distribution, interpret the posterior distribution, and check that the model is a reasonable description of the data.

\subsection{Data selection}

To reduce the number of uncertainties between the studied time periods, a volume was defined (Figure 1) from which the events were exported for the chosen time periods. The time periods were selected so that the production rate in the orebody was the same, although the production sequencing was different. It has been noted before that it is mucking and not blasting that controls the seismic activity in the crown pillar. The before-dataset contains 23,786 events and the after-dataset 20,569 events. These datasets are used as input to the modelling. There were a few sensors added to the system between the two time periods, which has been accounted for by using only events above the magnitude of completeness. This precaution eliminates the effects of change, such as the sensitivity of the system. When comparing the two time periods, there is no obvious migration of events, so the assumption of similar conditions holds true.

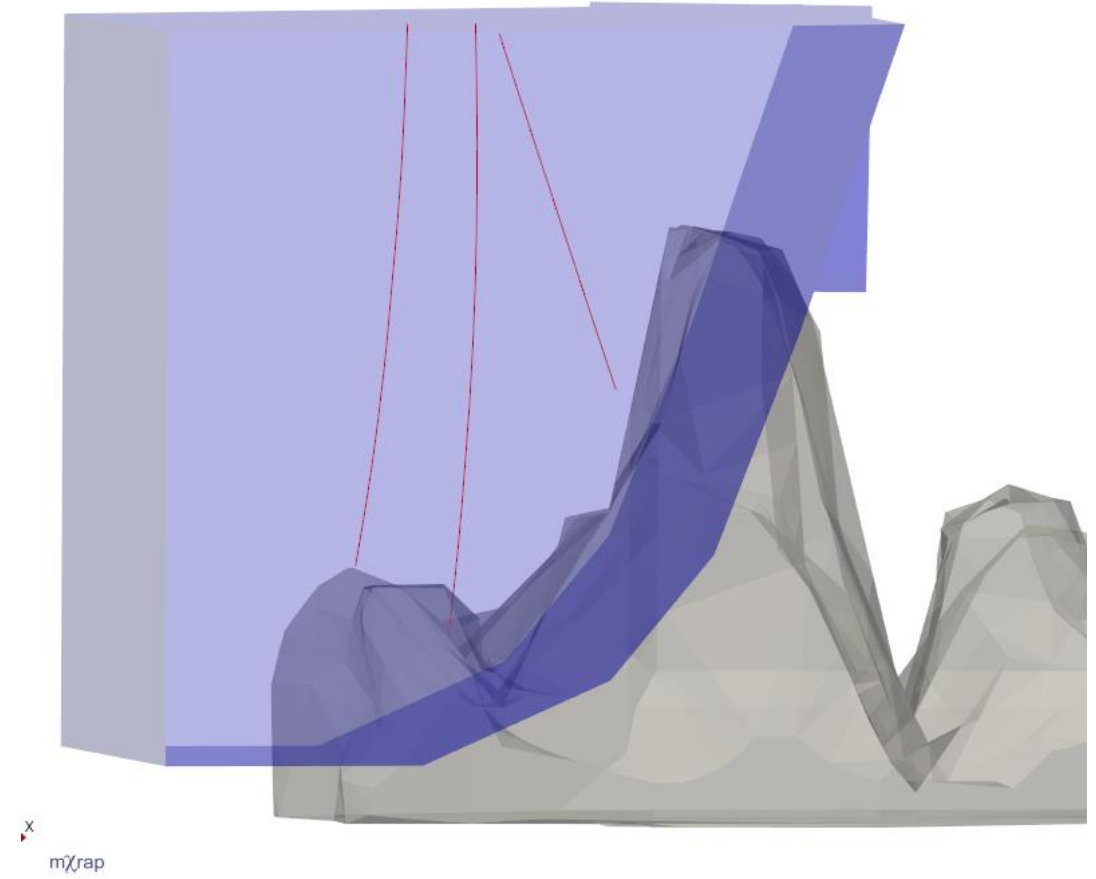

Figure 1 Studied volume (blue shape). The grey shape is an outline of the cave estimated from the seismicity, the red lines are the fracturing holes drilled from the ground surface

\subsection{Define a descriptive model}

Seismic data is often plotted in a Gutenberg-Richter (GR) diagram where the slope of the curve (the $b$-value) indicates the relative frequency of small and large events. A higher b-value means less extreme events can occur. In this study, we want to investigate if there is a difference in the b-value for the two time periods, before and after the hydraulic fracturing trial. The geographical spread of the geophones used for seismic monitoring means that the seismic system has varying sensitivity in different areas of the mine, and that the sensitivity changes over time as the system is expanded or geophones are lost. Small events have high frequencies and they are therefore often under-recorded by the seismic system, which means that the 
linear GR law does not hold for small magnitudes. This problem is usually handled by discarding all events of a magnitude lower than a specific threshold, called the magnitude of completeness $\left(m_{c}\right)$. The value of $m_{c}$ can be determined by visual inspection of the GR curve, or be provided by software such as $m$ Xrap (Harris \& Wesseloo 2015) (Figure 2). For this dataset (before fracturing), mXrap determines $m_{c}$ to be -1.6 , which leaves 12,679 out of 23,786 events in the dataset. This means that almost $50 \%$ of the total number of events are not used for analysis. Choosing $m_{\mathrm{c}}$ to be -1.7 gives 15,330 events in the dataset and $m_{\mathrm{c}}=-1.8$ gives 18,254 events. Determination of the b-value is very sensitive to the choice of $m_{c}$ (see e.g. Martinsson \& Jonsson 2018). For the dataset after fracturing, $m$ Xrap determines $m_{c}$ to be -1.8 (Figure 3 ).

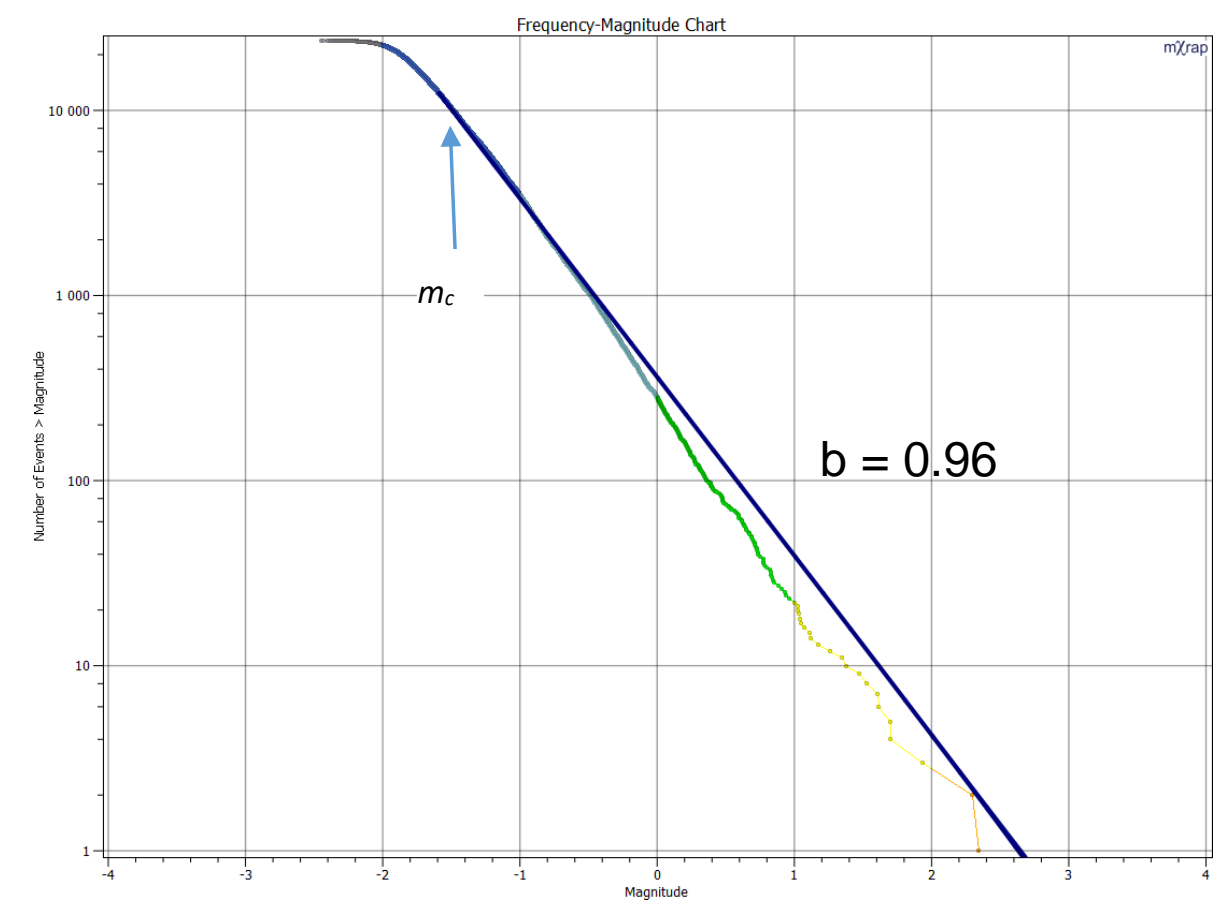

Figure 2 Frequency-magnitude plot showing the magnitude of completeness, $m_{c}$, and $b$-value for the before-fracturing events

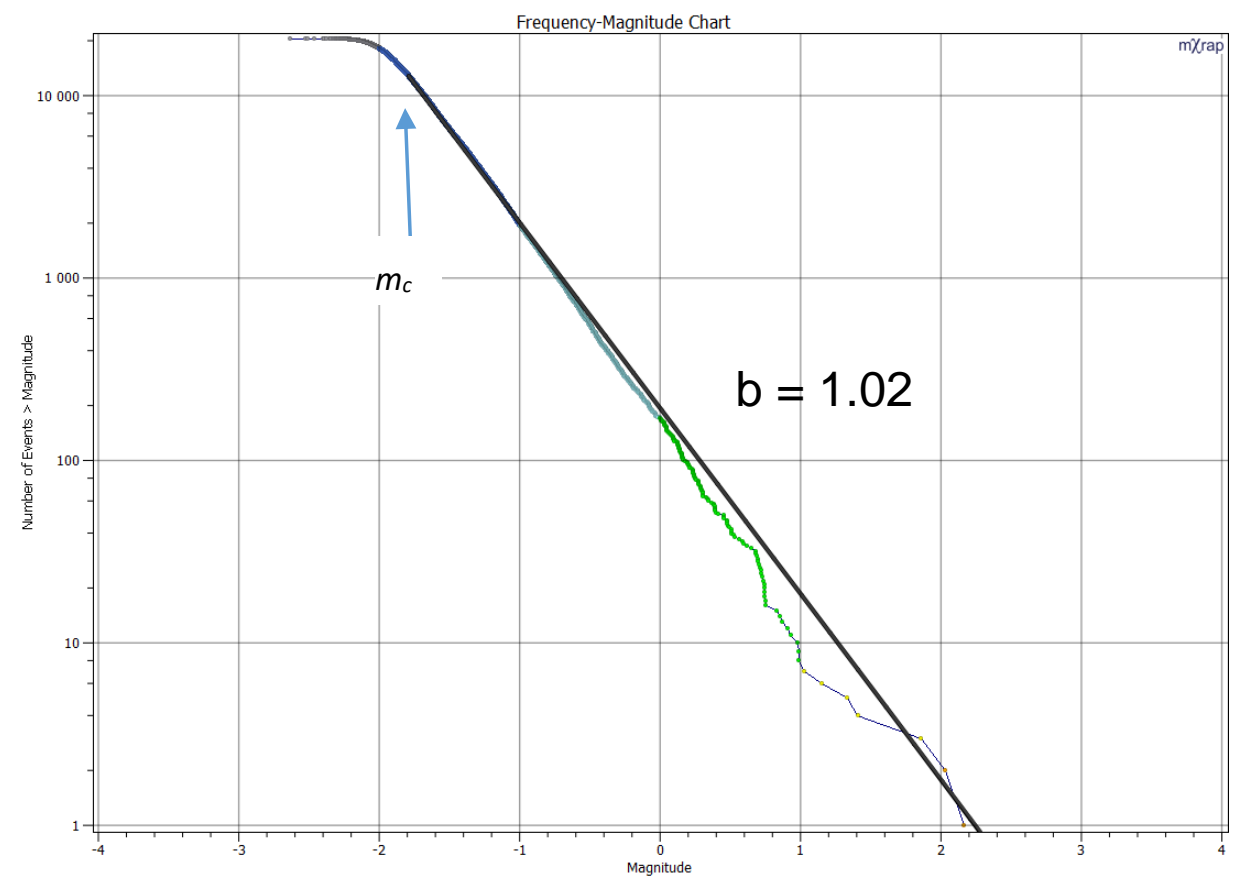

Figure 3 Frequency-magnitude plot showing the magnitude of completeness, $m_{c}$ and $b$-value for the after-fracturing events 
The GR curve is plotted on a log scale, which gives a linear behaviour above the magnitude of completeness $\left(m_{c}\right)$. Plotting the observed magnitudes without the log scale gives an exponential curve, which can be described by:

$$
N=10^{(a-b m)}
$$

where $N$ is the number of events having a magnitude $\geq m$, and $a$ and $b$ are parameters describing the activity and b-value, respectively.

Above the magnitude of completeness threshold, the magnitudes follow an exponential distribution.

$$
f(y, \lambda)=\lambda e^{-\lambda y}
$$

where $\lambda$ is the parameter, $y=m-m_{c}$ and the relationship to the b-value is

$$
b=\lambda / \ln (10)
$$

In this study, the comparison was done by adding a parameter $\Delta \lambda$ to the $\lambda$ of the after-fracturing group of data. If $\Delta \lambda$ is zero, there is no difference between the before and after groups of data, but if $\Delta \lambda$ is different from zero, there is a difference. A positive $\Delta \lambda$ indicates that $\lambda$, and hence the $b$-value, has increased for the after group.

The analysis was done using PyStan (PyStan 2018), where Stan (STAN 2018) is a statistical package, and $\mathrm{Py}$ is short for the programming language Python. Stan uses Hamiltonian Monte Carlo simulation which makes it more effective for large or complex samples compared to other samplers. In Stan, the model is built up according to a fixed structure. Figure 4 illustrates the Bayesian statistical model using the graphical model representation proposed by Kruschke (2015). The data, $y_{i}=m_{i}+m_{\mathrm{c}}$, consist of magnitude values of events of events exceeding $m_{\mathrm{c}}$ from before and after the fracturing trial, to which the value of magnitude of completeness has been added to shift the distribution along the $x$-axis. The data is modelled by an exponential distribution, whose shape is determined by $\lambda$ and the possible difference $\Delta \lambda$. The possible difference $\Delta \lambda$ between the groups is multiplied by a time period index (s[i]) depending on whether the events belong to the before-fracturing group $(s[i]=0)$ or the after-fracturing group $(s[i]=1)$, for the ith magnitude.

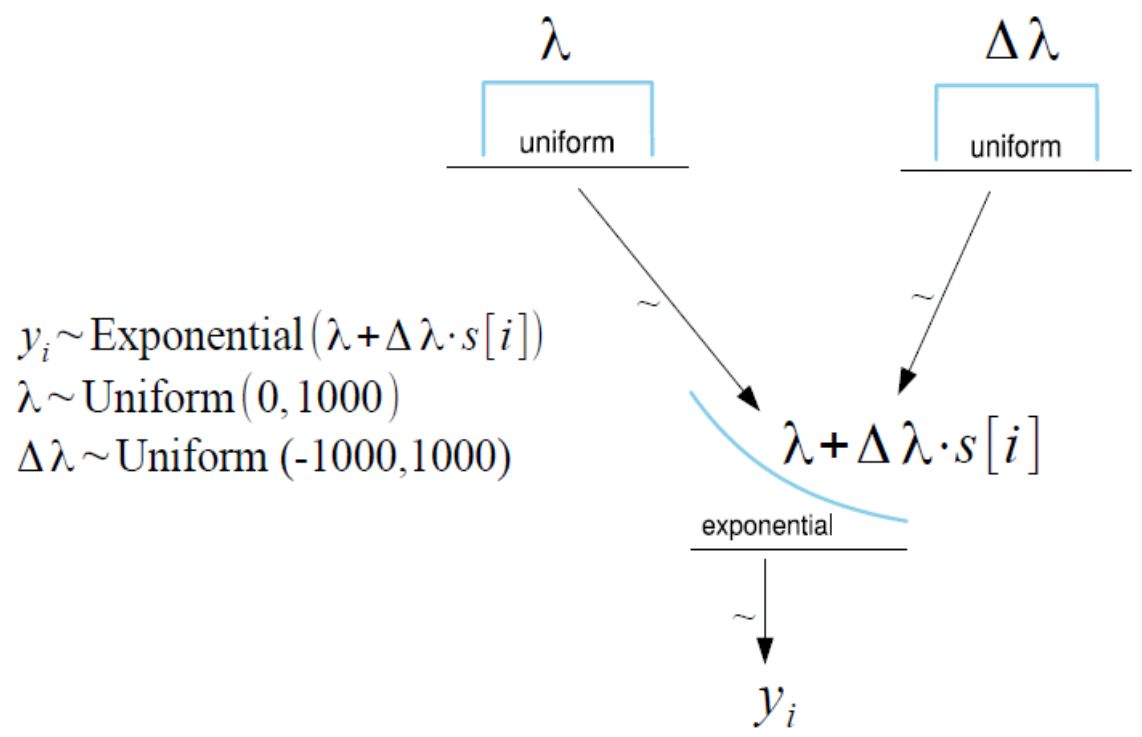

Figure 4 Bayesian statistical model of the dependencies between the data and the parameters. The data is truncated at $m_{c}$ and then translated as $y_{i}=m_{i}+m_{c}$. so that a standard exponential distribution can be applied in Stan

The magnitude of completeness $\left(m_{c}\right)$ was chosen to be -1.7 to ensure enough recorded events were used to inform the model. The model was set to run a chain of 100,000 samples. 


\subsection{Specify prior}

In modelling, the choice of prior can have a great influence on the results. A vague prior distribution means the data has a greater influence on the posterior distribution than the prior, while an informed (well-defined and specific) prior gives the opposite. Prior distributions have to be assigned for the two parameters $\lambda$ and $\Delta \lambda$. In this model, the prior distribution for $\lambda$ has been assumed to be a uniform distribution between 0 and 1,000, with the restriction that the $\lambda$ has to be greater than zero. The prior for $\Delta \lambda$ is also assumed to be a uniform distribution that can have values between $-1,000$ and 1,000 .

\subsection{Posterior distribution}

Running the model produces posterior distributions for the two groups, before and after, as well as credible values of the parameters $\lambda$ and $\Delta \lambda$. A way to check the uncertainty of the parameter values is to determine the span of values that are most credible and cover $95 \%$ of the distribution (Kruschke 2015). This is called the highest density interval (HDI). Another important check of the model is the effective sample size (ESS), which indicates how stable and accurate the representation of posterior distribution is based on the samples generated. Out of 100,000 posterior samples using Stan, the ESS for both $\lambda$ and $\Delta \lambda$ are above 45,000. Kruschke (2015) recommend that the ESS should be at least 10,000 to get an accurate determination of the $95 \% \mathrm{HDI}$.

\section{Results and discussion}

The output from the model is posterior distributions for all parameters. All results discussed here are in relation to the chosen magnitude of completeness, $m_{c}=-1.7$. The results are shown in terms of $b$-value, which has been determined from Equation 3. The sensitivity of the results to the choice of magnitude of completeness is also discussed.

\subsection{Evaluation of model}

The difference between the groups is shown by the parameter $\Delta b$. The posterior distribution for $\Delta b$ is shown in Figure 5 . The mean value of $\Delta b$, for $m_{c}=-1.7$ is 0.096 , and the $95 \% \mathrm{HDI}$ interval is 0.072 to 0.121 , which clearly excludes zero.

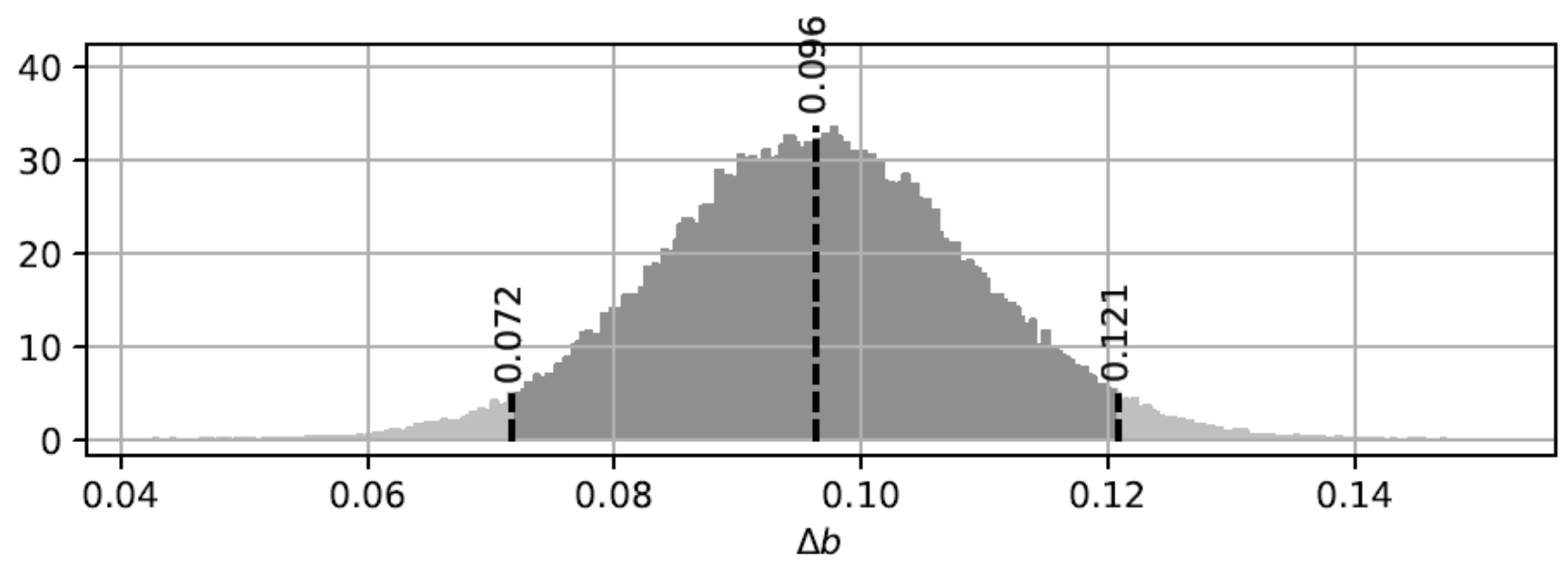

Figure 5 Posterior distribution of $\Delta b=\Delta \lambda / \ln (10)$ for $m_{c}=-1.7$

The effect on the b-value is shown in Figure 6. The mean of the b-value for the before fracturing events is 0.928 with the $95 \% \mathrm{HDI}$ ranging from 0.913 to 0.942 . The mean of the b-value for the after-fracturing events is 1.024 with the $95 \% \mathrm{HDI}$ ranging from 1.004 to 1.044 . 


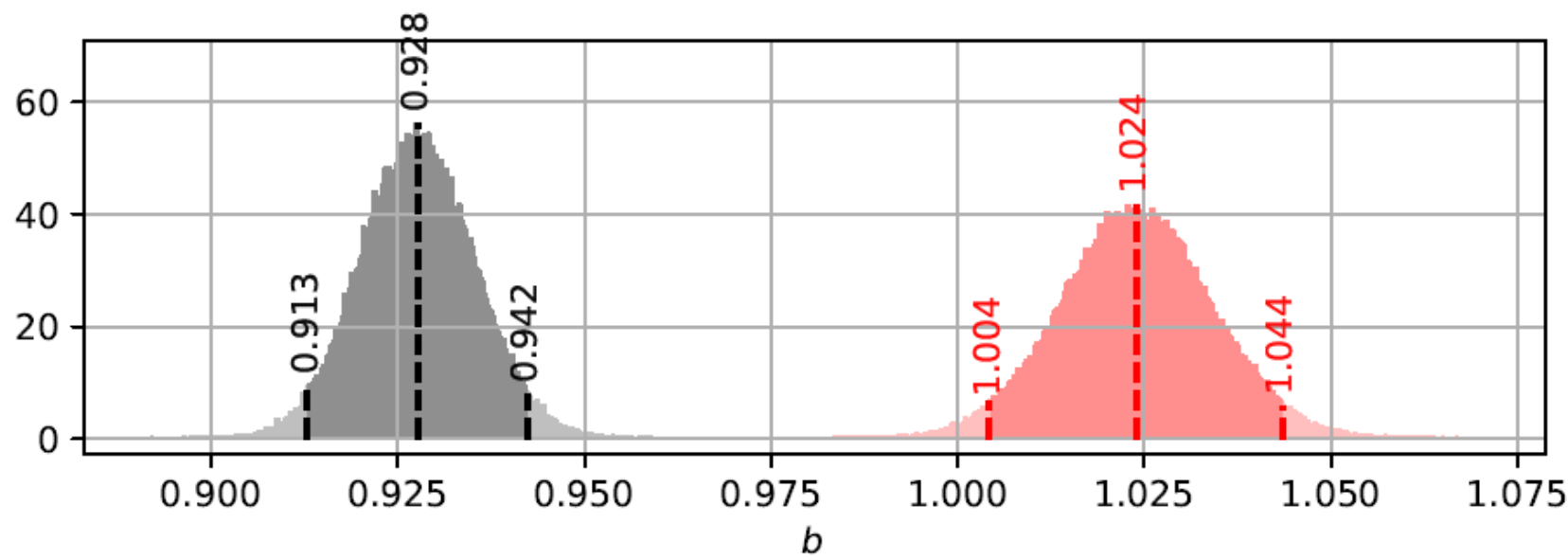

Figure 6 Posterior distribution of b-values before (black) and after (red) including lines indicating mean and $95 \%$ HDI limits

Figure 7 shows the exponential distribution for the before (black) and after (red) groups, plotted together with the corresponding histograms of the input data (dots). The two panels are identical except for the difference in vertical scale. The log-scale representation clearly shows a difference in slope of the two groups. The curves in corresponding colour represent the estimated exponential distribution of the two groups. The thin lines are credible distribution curves generated using a random subset of 1,000 posterior samples from the histogram in Figure 6 , and the bold lines mark the distribution using the sample mean.
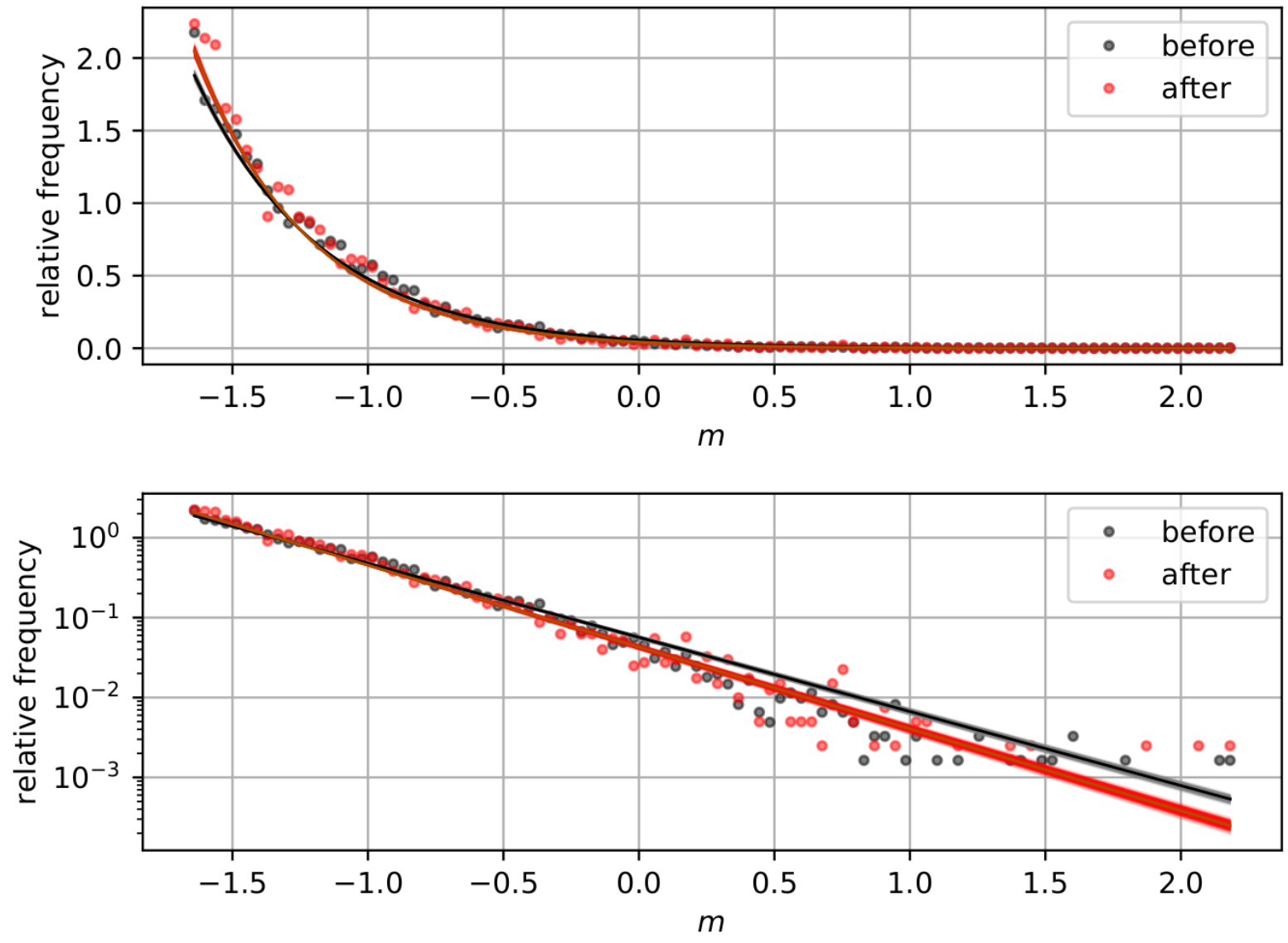

Figure 7 Exponential distribution for the frequency of events-magnitude relationship before and after the trial (lines), plotted together with measured data (dots). The bold lines represent the exponential distribution using the corresponding mean b-value of the two groups in Figure 6 , and the several thin lines surrounding the bold line are credible distributions using a subset of 1,000 random samples from the posterior distributions in Figure 6 
The exponential distributions based on a random subset of credible parameter values show minor deviation from the distributions obtained using the sample means in both groups. The effect of the estimation uncertainty of the b-value is best spotted in log scale at larger magnitudes. To conclude, we can say that the posterior uncertainty of the b-value shown in Figure 6 has minor impact on the deviation of the GR curve.

Note that Figures 2 and 3 are cumulative histograms and Figure 7 is plain histograms of the magnitudes with bin size 0.1. The cumulative operator in Figures 2 and 3 will accumulate all errors starting from the left, and hence more errors are summed up towards the right of the data in the figures. The effect of this accumulation of errors is shown as a 'random walk' behaviour of the data in these figures, and hence we should be careful when drawing conclusions regarding the validity of the GR model based on cumulative histograms.

Conclusions regarding the validity of the GR model to the data should instead be based on the non-cumulative histograms shown in Figure 7, and specifically in linear vertical scale (i.e. the top panel in Figure 7). When drawing conclusions based on the bottom panel in logarithmic scale, we must have the logarithmic scale in mind.

\subsection{Sensitivity of model}

The same model was run for different values of $m_{\mathrm{c}}$ to determine the effect on b-value and $\Delta b$. Figure 8 shows $b$-values before and after the fracturing trial for a range of magnitude of completeness between -1.9 and -1.4. For this range of $m_{c}$, there is a difference in b-value for all $m_{c}$ tested, and the $95 \% \mathrm{HDI}$ for the before and after value does not overlap for any $m_{c}$, indicating that hydraulic fracturing has the desired effect on the b-value at reasonable values of $m_{c}$.

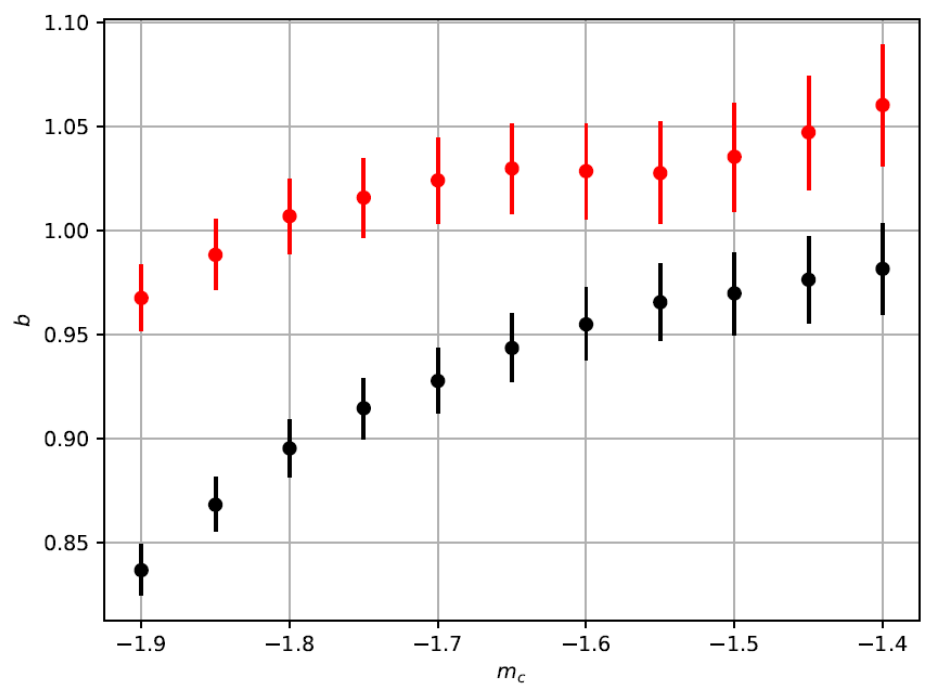

Figure 8 Difference in b-value before (black dots) and after (red dots) fracturing trial for $-1.9<m_{c}<-1.4$, also showing $95 \% \mathrm{HDI}$ as vertical lines

Figure 9 shows $\Delta \mathrm{b}$-values for different values of magnitude of completeness. The smallest difference occurs for $m_{c}=-1.55$, but for no choice of $m_{c}$ does the HDI of $\Delta b$ include zero. It can also be noted that for low $m_{c}$, the HDI is smaller than for large $m_{c}$. This increased uncertainty in the estimate is caused by the fact that a smaller dataset is used to inform the model than for smaller $m_{\mathrm{c}}$. 


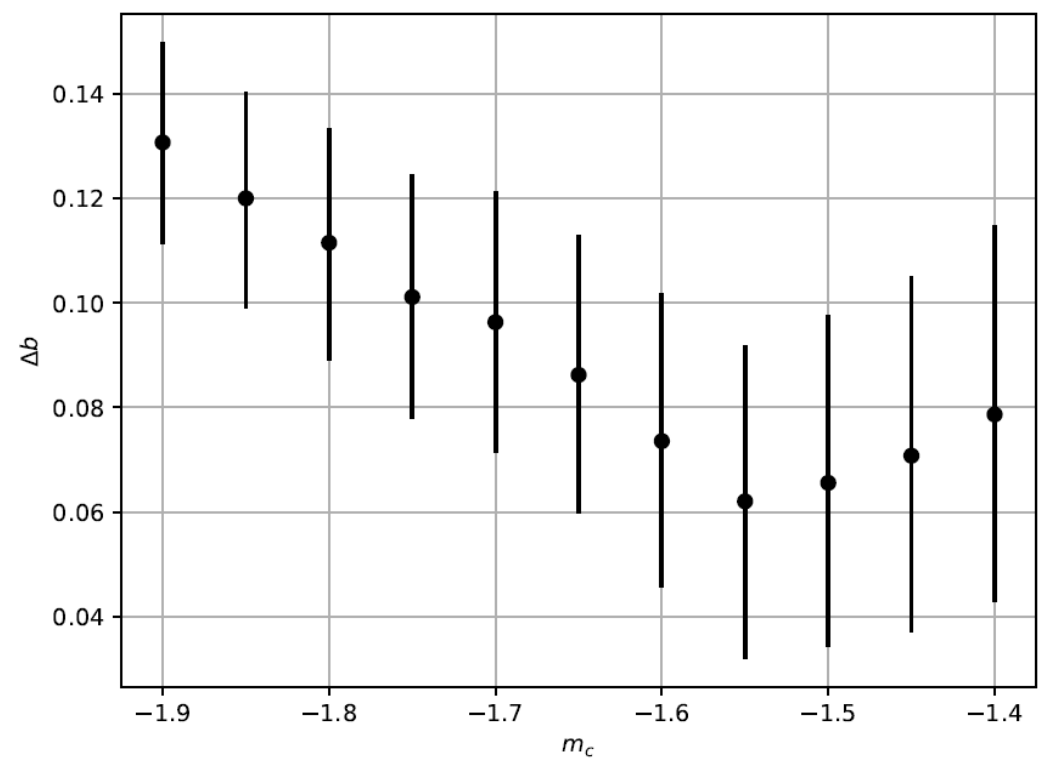

Figure 9 Difference in $\Delta b=\Delta \lambda / \ln (10)$ for $-1.9<m_{c}<-1.4$, also showing $95 \% \mathrm{HDI}$ as vertical lines

In summary, the analysis shows that there is a difference in seismic behaviour when studying the time periods before and after the hydraulic fracturing trial. The sensitivity analysis with regard to choice of magnitude of completeness suggests that this value should be chosen with care. Regardless of choice of $m_{c}$, the $95 \% \mathrm{HDI} \Delta b$ (or equivalently $\Delta \lambda$ ) did not in any case include zero, so the conclusion that there is a difference is valid.

\section{Conclusion}

The $95 \% \mathrm{HDI}$ for $\Delta b$ did not include zero for any value of $m_{\mathrm{c}}$. This gives confidence in stating that there is a difference in the seismic behaviour of the crown pillar after the hydraulic fracturing trial. Some large seismic events are still expected to happen, but not as frequently as before. The magnitude of completeness has an effect on the results. The choice of $m_{\mathrm{c}}=-1.7$ is a compromise that gives a reasonably large dataset to inform the model, reasonable accuracy in the result, and gives reasonable computational time.

\section{$5 \quad$ Recommendations}

Since the estimation of the b-value is sensitive to the choice of magnitude of completeness, analysis of the same dataset should be done using the method outlined in Martinsson and Jonsson (2018) where all events are included. That would give a more robust estimation of the b-value. The analysis should be repeated when a larger tonnage has been mucked from the drifts below the hydraulically fractured volume in the crown pillar. Only then can the question of whether a large enough volume was fractured in the trial be answered. Another study that could be conducted is if the relationship between production and the seismic activity in the crown pillar has changed after the hydraulic fracturing trial.

\section{References}

Harris, PC \& Wesseloo, J 2015, mXrap, computer software, Australian Centre for Geomechanics, The University of Western Australia, Perth, https://www.mXrap.com

Kruschke, JK 2015, Doing Bayesian Data Analysis: A Tutorial with R, JAGS, and Stan, 2nd edn, Academic Press, Cambridge.

Lowther, RJ, Olivier, L, Lett, JL \& Brunton, I 2016, 'Implementation of a surface-based hydraulic fracturing program to successfully propagate a large cave through hard, competent near-surface rock masses to achieve breakthrough', Proceedings of MassMin 2016, The Australasian Institute of Mining and Metallurgy, Melbourne, pp. 83-95.

Martinsson, J \& Jonsson, A 2018, 'A new model for the distribution of observable earthquake magnitudes and application to b-value estimation', IEEE Geoscience and Remote Sensing Letters, vol. 15, no. 6, https://dx.doi.org/10.1109/LGRS.2018.2812770

PyStan 2018, PyStan, computer software, https://pystan.readthedocs.io/en/latest/index.html

Stan 2018, Stan, computer software, http://mc-stan.org 